\title{
Photometric Properties of White Dwarf Dominated Halos
}

\author{
Hyun-chul Lee ${ }^{1,3}$, Brad K. Gibson $^{1}$, Yeshe Fenner ${ }^{1}$, Chris B. Brook ${ }^{1}$, Daisuke Kawata ${ }^{1}$, \\ Agostino Renda $^{1}$, Janne Holopainen ${ }^{1,2}$, and Chris Flynn ${ }^{1,2}$ \\ ${ }^{1}$ Centre for Astrophysics and Supercomputing, Swinburne University, Melbourne VIC 3122, Australia \\ ${ }^{2}$ Tuorla Observatory, Piikkiö FIN-21500, Finland \\ ${ }^{3}$ E-mail: hclee@astro.swin.edu.au
}

Received 2003 December 29, accepted 2004 January 13

\begin{abstract}
Using stellar population synthesis techniques, we explore the photometric signatures of white dwarf progenitor dominated galactic halos, in order to constrain the fraction of halo mass that may be locked up in white dwarf stellar remnants. We first construct a $10^{9} \mathrm{M}_{\odot}$ stellar halo using the canonical Salpeter initial stellar mass distribution, and then allow for an additional component of low- and intermediate-mass stars, which ultimately give rise to white dwarf remnants. Microlensing observations towards the Large Magellanic Cloud, coupled with several ground-based proper motion surveys, have led to claims that in excess of $20 \%$ of the dynamical mass of the halo $\left(10^{12} \mathrm{M}_{\odot}\right)$ might be found in white dwarfs. Our results indicate that (1) even if only $1 \%$ of the dynamical mass of the dark halo today could be attributed to white dwarfs, their main sequence progenitors at high redshift $(z \approx 3)$ would have resulted in halos more than 100 times more luminous than those expected from conventional initial mass functions alone, and (2) any putative halo white dwarf progenitor dominated initial mass function component, regardless of its dynamical importance, would be virtually impossible to detect at the present day, due to its extremely faint surface brightness.
\end{abstract}

Keywords: galaxies: halos — galaxies: luminosity function, mass function - galaxies: stellar content white dwarfs

\section{Introduction}

The flat rotation curves of spiral galaxies suggest that their galactic halos are mostly composed of dark matter (Rubin et al. 1980). The precise nature of this dark matter remains an open question, one with important implications for cosmology and galaxy formation. Observations of microlensing events from programs such as MACHO and EROS suggest that perhaps of order $20 \%$ of the Galactic halo may be composed of $\sim 0.5 \mathrm{M}_{\odot}$ compact objects (Alcock et al. 2000; Afonso et al. 2003). One particularly appealing source of $0.5 \mathrm{M}_{\odot}$ objects that was immediately vetted in the literature was that of a population of faint, old, white dwarfs. ${ }^{1}$

Alcock et al. (2000) analysed 5.7 years of photometric data on stars in the Large Magellanic Cloud, in search of gravitational microlensing events. They concluded that the fraction of massive compact halo objects (MACHOs) is about $20 \%$ (with an average mass of $0.5 \mathrm{M}_{\odot}$ per MACHO), and that the total mass in MACHOs out to $50 \mathrm{kpc}$ is $10^{11} \mathrm{M}_{\odot}$, assuming the lenses are located in the Galactic halo. ${ }^{2}$ The recent EROS data toward the Small Magellanic Cloud allow for a maximum of $\sim 25 \%$ of a spherical, isothermal, and isotropic Galactic halo of mass $4 \times 10^{11} \mathrm{M}_{\odot}$ out to $50 \mathrm{kpc}$ which could be composed of

\footnotetext{
1 'Appealing' in the sense that they are relatively 'mundane' astronomical objects, obviating the need to draw upon more exotic solutions.

${ }^{2}$ Not necessarily a unanimously accepted hypothesis, e.g. Sahu \& Sahu (1998); Di Stefano (2000).
}

objects with mass between $2 \times 10^{-7}$ and $1 \mathrm{M}_{\odot}$ (Afonso et al. 2003). The absence of events with crossing times shorter than 10 days and the lack of sufficient numbers of low-mass main sequence stars in the Hubble Deep Field essentially rules out planet-like objects and brown dwarfs as the microlens candidates (Gould et al. 1998; Lasserre et al. 2000). The current favoured mass range from the microlensing experiments also rules out the remnants of more massive stars, including neutron stars and black holes, as potential (and substantial) baryonic dark matter candidates.

A rich literature has emerged over the past five years exploring the pros and cons of the hypothesis that white dwarfs may comprise a significant fraction of the dynamical mass of galaxies. Ryu et al. (1990) constructed a simple galactic halo chemical evolution model and ruled out neutron stars, but not white dwarfs, as dark matter candidates, based on metallicity and luminosity considerations. The low incidence of Type Ia supernovae observed in the outer regions of galaxies lead Smecker \& Wyse (1991) to impose tight upper limits on the fraction of white dwarfs in galactic halos. Charlot \& Silk (1995), using population synthesis models coupled with number counts of faint galaxies in deep galaxy surveys, showed that only a small fraction $(\lesssim 10 \%)$ of present-day halos could be in the form of white dwarfs. Madau \& Pozzetti (2000) showed that in order to avoid the overproduction of extragalactic background light, the halo white dwarf mass fraction should be $\lesssim 5 \%$. 


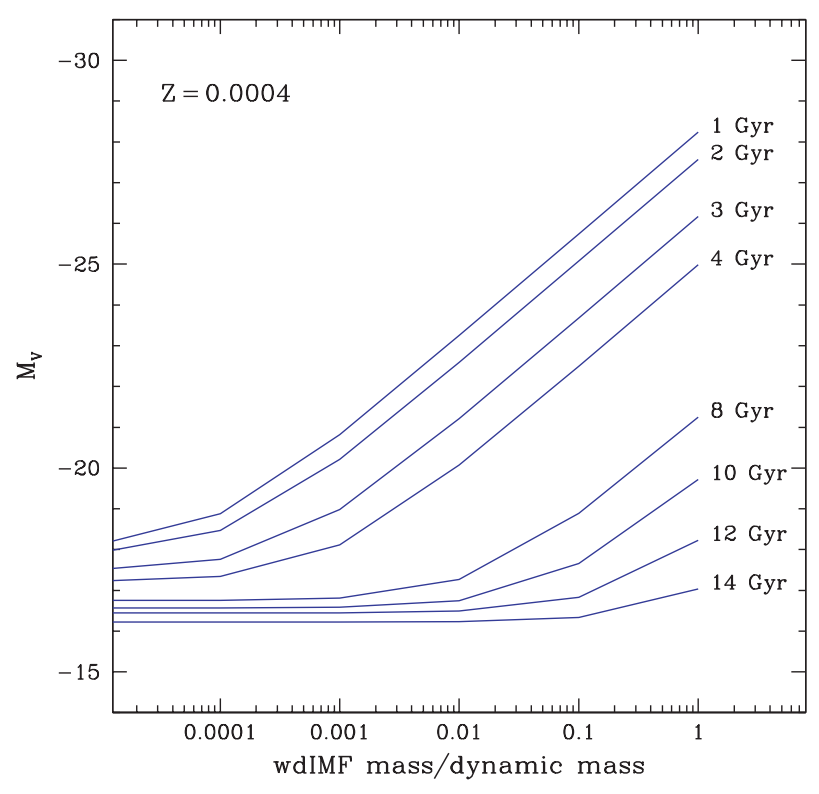

Figure 1 The absolute $\mathrm{V}$ band magnitude as a function of halo white dwarf mass fraction for eight ages ranging from 1 to $14 \mathrm{Gyr}$. The left-hand limit to the plot corresponds to the minimum contribution due to the known $10^{9} \mathrm{M}_{\odot}$ stellar halo intrinsic to spirals such as the Milky Way (our default model). Passive evolution of this basic system would be $\sim 2$ magnitudes brighter at redshift $z \sim 3$ (an age of $\sim 1 \mathrm{Gyr}$, for a redshift of formation of five). Conversely, a $10^{12} \mathrm{M}_{\odot}$ halo comprised of nothing but white dwarfs at the present day would have been $\sim 10$ magnitudes brighter at $z \sim 3$.

Gibson \& Mould (1997) and Brook et al. (2003) placed more severe constraints upon the halo white dwarf mass fraction $(\lesssim 1-2 \%)$ by using the observed carbon, nitrogen, and oxygen abundance patterns of halo stars.

Following Larson's (1986) suggestion that a remnantdominated form of the initial mass function (IMF) could account for the unseen mass in the solar neighbourhood, both Chabrier et al. (1996) and Adams \& Laughlin (1996) devised physically motivated models allowing for a white dwarf progenitor dominated IMF, consistent with the aforementioned MACHO and EROS microlensing results. Such an IMF differs from that of the classical Salpeter (1955) functional form through the absence of both low mass $\left(\lesssim 1 \mathrm{M}_{\odot}\right)$ and high mass $\left(\lesssim 6 \mathrm{M}_{\odot}\right)$ stars (Gibson \& Mould 1997; Figure 1).

In this paper, we address explicitly the photometric properties of the luminous early phases of putative white dwarf progenitor dominated galactic halos, in addition to their temporal evolution. In Section 2, we describe our stellar population models and the different functional forms for the IMFs considered here. The results of our calculations are then presented in Section 3, with the accompanying discussion and conclusions provided in Section 4.

\section{Models}

Using our evolutionary stellar population synthesis code (Lee et al. 2002), we have calculated the evolution of the photometric properties of galactic halos as a function of the dynamical mass fraction tied up in putative populations of white dwarfs. We allow the total present-day mass fraction of white dwarfs to range from 0 to $100 \% 3$ of the halo's dynamical mass, which is taken for this exercise to be $10^{12} \mathrm{M}_{\odot}$ (after that of the Milky Way — Fich \& Tremaine 1991). All models described herein contain a standard $10^{9} \mathrm{M}_{\odot}$ stellar component, ${ }^{4}$ which is itself described by the Salpeter IMF (by number):

$$
\Phi(m) \mathrm{d} m=\mathrm{d} n / \mathrm{d} m=A m^{-x} \mathrm{~d} m,
$$

with $x=2.35$.

The fraction of halo dark matter contained in white dwarfs is then determined by the amount of matter contained in a supplemental IMF described by a truncated power law of the form

$$
\Phi(m) \mathrm{d} m=\mathrm{d} n / \mathrm{d} m=A e^{-(\bar{m} / m)^{\beta}} \times m^{-\alpha} \mathrm{d} m
$$

for which we use $\bar{m}=2.7, \beta=2.2$, and $\alpha=5.75$ (Chabrier et al. 1996). The peak of this skewed-Gaussian functional form for the IMF (hereafter, wdIMF) occurs at $m \approx 2 \mathrm{M}_{\odot}$, favouring the production of white dwarf progenitors. The wdIMF yields a present-day Galactic halo mass-to-light ratio $\gg 100$ after a Hubble time as most of its initial stellar distribution has since become very faint remnants.

The stellar population synthesis models presented here are based upon the $Y^{2}$ isochrones ${ }^{5}$ (Kim et al. 2002) with $[\alpha / \mathrm{Fe}]=+0.3$, coupled to the post-red giant branch stellar evolutionary tracks of Yi et al. (1997). We have calibrated the horizontal-branch morphology with the Milky Way globular clusters, as in Lee et al. (2000, 2002). The stellar library of Lejeune et al. (1998) was taken for the conversion from theoretical to observable quantities. In our calculations, the stellar remnant mass was assumed to be $0.5 \mathrm{M}_{\odot}$ for initial stellar masses below $8 \mathrm{M}_{\odot}$, and $1.0 \mathrm{M}_{\odot}$ for initial stellar masses in excess of $8 \mathrm{M}_{\odot}$.

New cooling models for white dwarfs have become available recently which possess somewhat different behaviour in the colour-magnitude plane at advanced ages (e.g. Hansen 1998; Richer et al. 2000), when compared with the conventional models adopted in most population synthesis codes (including ours). While we have experimented with their inclusion, due to the extremely low luminosity at which these new models diverge from the classical ones (at $M_{V} \sim 17.5$, after $\sim 8$ Gyr of cooling), our results are not seriously impacted by the choice of specific white dwarf cooling tracks.

The following section presents results for our default model — a Milky Way-like halo with metallicity $[\mathrm{Fe} / \mathrm{H}] \sim-1.8$ (Ryan \& Norris 1991) — we also touch briefly upon the implications for more metal-rich halos such as that for M31.

\footnotetext{
${ }^{3}$ Strictly speaking, we allow up to $99.9 \%$ of the present-day mass fraction of white dwarfs as a $10^{9} \mathrm{M}_{\odot}$ stellar halo with the canonical Salpeter IMF providing the base for our model.

${ }^{4} \mathrm{~A}$ stellar mass of $10^{9} \mathrm{M}_{\odot}$ for the Milky Way halo is reasonable once one adopts, for example, a surface density profile proportional to $\mathrm{r}^{-2}$ normalised by the local stellar halo density, e.g. Preston et al. (1991). 5 http://csaweb.yonsei.ac.kr/ kim/yyiso.html
} 


\section{Results}

Figure 1 shows the variation in absolute $\mathrm{V}$ band magnitude as a function of halo dynamical mass fraction tied up in white dwarfs, for ages ranging from 1 to $14 \mathrm{Gyr}$. The asymptotic values of $M_{V}$ approached for mass fractions $<10^{-4}$ simply reflect the model's assumed base $10^{9} \mathrm{M}_{\odot}$ Salpeter IMF stellar component; ${ }^{6}$ a mass fraction of unity corresponds to a $10^{12} \mathrm{M}_{\odot}$ halo comprised entirely (essentially) of white dwarfs. After $\sim 13 \mathrm{Gyr}$, a $100 \%$ white dwarf halo is, for all intents and purposes, indistinguishable photometrically from a halo containing no remnants aside from the underlying population attributed to the (known) Salpeter-like component. For younger stellar systems though, white dwarf progenitors preferentially populate the main sequence, giant branch, and horizontal branch phases, and rapidly dominate the luminosity of the halo.

Although unlikely, if the total dynamic mass were composed of white dwarf remnants, this halo would have been $\sim 10$ magnitudes brighter $\sim 1$ Gyr after its 'formation' (i.e. $z \sim 3$, for a redshift of formation of five). In comparison, the same halo (by dynamical mass), but now with only the known $10^{9} \mathrm{M}_{\odot}$ Salpeter IMF stellar component, would only be $\sim 2$ magnitudes brighter at the same lookback time. Even if only $1 \%$ of the mass of the dark halo was in the form of this wdIMF component, it would have been a factor of $\sim 100$ times more luminous than the canonical halo at the same redshift $z \sim 3$.

Figure 2 presents the dependence of colour $(V-I)$ on the fraction of the dynamic halo mass in the form of white dwarf remnants for halos ranging in age from 1 to 14 Gyr. A white dwarf dominated halo is indistinguishable in colour from a Salpeter-only halo for lookback times $\lesssim 8 \mathrm{Gyr}$ (note the essentially flat behaviour of the curves as a function of wdIMF). ${ }^{7}$

The only marginal difference between the two scenarios occurs at ages corresponding to redshifts in the range $1 \lesssim z \lesssim 2-$ for instance, if $1 \%$ of the dark halo mass was in the form of white dwarfs, its stellar component would have been $\sim 0.08$ magnitude redder than a Salpeter-only halo (left-hand limit of Figure 2) at early epochs because of the former's more significant populations of red giants and red horizontal-branch stars.

\footnotetext{
${ }^{6}$ We re-emphasise here that the asymptotic value of $M_{V}$ approached at low white dwarf mass fractions in Figure 1 corresponds to the values expected for a halo with a base stellar component of mass $10^{9} \mathrm{M}_{\odot}$, constructed with a conventional Salpeter IMF — representative of what one might expect for a canonical model of the Milky Way. This absolute magnitude would of course change should a different 'base' Salpeter IMF halo component be employed - for example $M_{V}$ for a halo of the same metallicity would be 2.5 magnitudes brighter should the base stellar halo component have a mass of $10^{10} \mathrm{M}_{\odot}$. Furthermore, the halo would be about 1 magnitude fainter if its metallicity was solar, as the main-sequence turnoff is fainter for metal-rich systems. Regardless, our analysis remains valid, as it is the differential between the models which is important, and not the 'zero-point' of the curves in Figure 1.

${ }^{7}$ The abrupt shift to significantly bluer colours for the $1 \mathrm{Gyr}$ isochrone is due to the treatment of convective core overshooting in the stellar models (Figure 6 in Yi 2003).
}

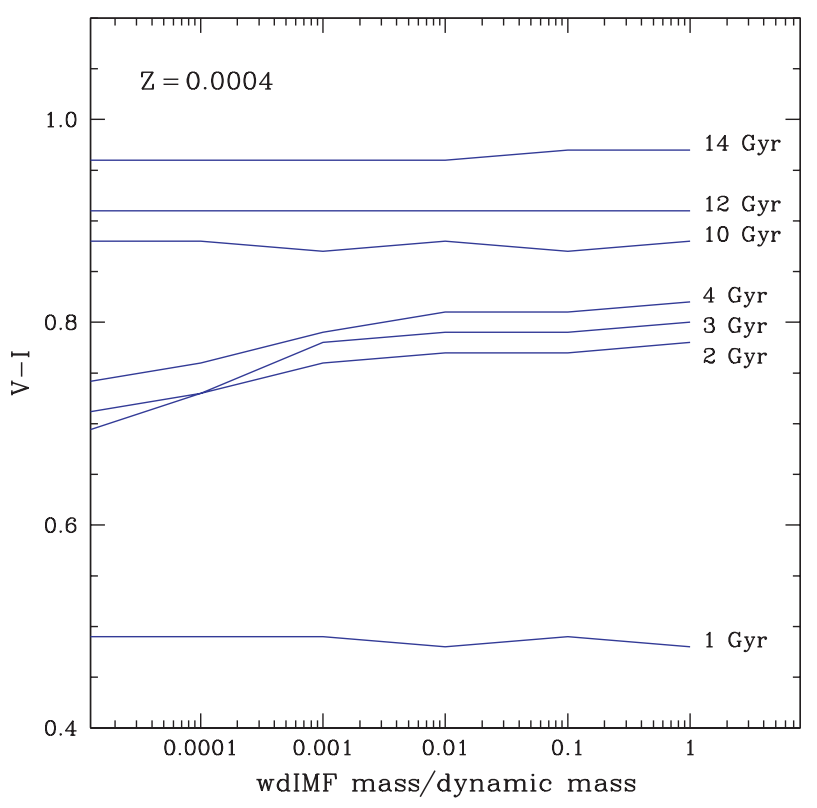

Figure 2 As in Figure 1, but now showing the variation in integrated colour $(V-I)$ as a function of halo white dwarf mass fraction.

The predicted colours in Figure 2 are also, of course, different for different metallicities. In general, the more metal-rich a halo is, the redder its stellar population. For a halo with a metallicity like that of M31 $([\mathrm{Fe} / \mathrm{H}] \sim-0.5$; Durrell et al. 2001), we note in passing that if $1 \%$ of the halo's dynamical mass were attributed to a wdIMF component, the $V-I$ colour at $1 \mathrm{Gyr}$ would be 0.55 , at $3 \mathrm{Gyr}$ it would be 1.14 , and at $14 \mathrm{Gyr}$ it would be 1.26 .

\section{Discussion}

In Figure 3 (after Chabrier 2001), we speculate on the surface brightness radial profile of a $10^{12} \mathrm{M}_{\odot}$ halo with a $10 \%$ (by mass) wdIMF component, but without the 'base' $10^{9} \mathrm{M}_{\odot}$ Salpeter IMF stellar component. ${ }^{8} \mathrm{We}$ have adopted a dark matter halo density profile of $\rho(r) \propto 1 / r^{2}$. For the surface mass density of the halo, we assumed $23 \mathrm{M}_{\odot} \mathrm{pc}^{-2}$ at $8 \mathrm{kpc}$ (Kuijken \& Gilmore 1991). It can be seen from Figure 3 that if $10 \%$ of the dark halo mass were composed of a wdIMF stellar component, its predicted surface brightness would be essentially undetectable. Put into context, the central surface brightness of the low surface brightness galaxy Malin 1 is $\mu_{0}(\mathrm{~V}) \sim 25.5$ (Bothun et al. 1987), i.e. this $10 \%$ wdIMF component lies 7-10 magnitudes fainter than the surface brightness of Malin 1!

Table 1 lists the surface brightness of such a galactic halo at a galactocentric radius of $60 \mathrm{kpc}$ for several passbands at a given age and wdIMF mass fraction. We do not wish to belabour the result here, and simply note that the surface brightnesses in the outer regions of these putative WD-dominated halos would be essentially

${ }^{8}$ By eliminating the underlying trace (by mass, not by luminosity) Salpeter component, we serve to emphasise the light contribution from the wdIMF component alone. 


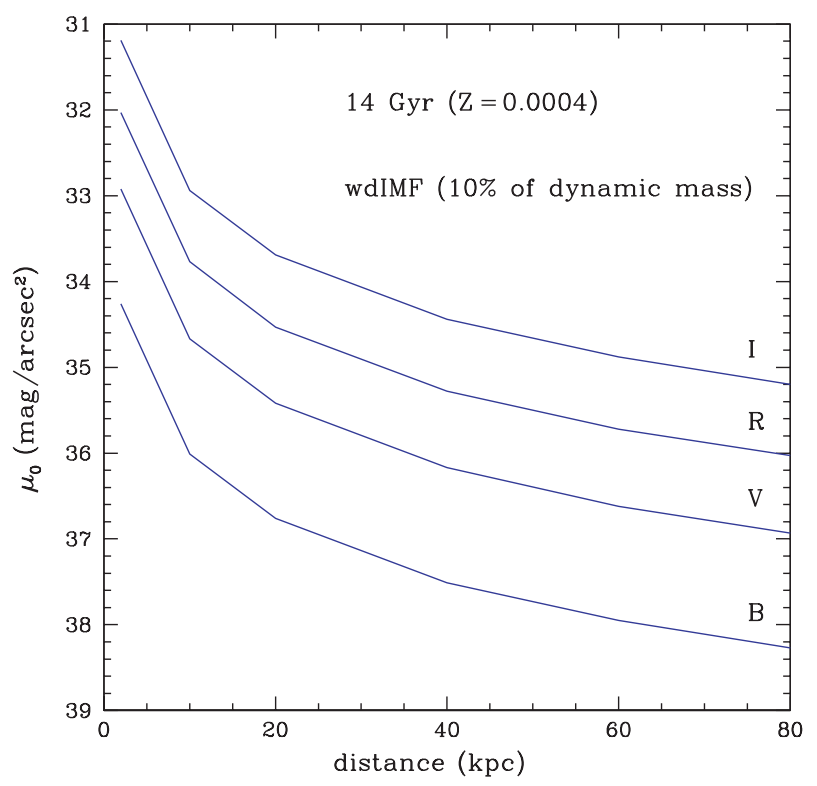

Figure 3 Surface brightness radial profile of a galactic halo with $10 \%$ of its dynamical mass comprised of a wdIMF stellar component, but now without the base $10^{9} \mathrm{M}_{\odot}$ Salpeter IMF stellar component, to emphasise the essentially undetectable wdIMF component's surface brightness (after Chabrier 2001).

Table 1. Surface brightness at $60 \mathrm{kpc}(Z=\mathbf{0 . 0 0 0 4})$

\begin{tabular}{lcccc}
\hline Age (wdIMF) & B & V & R & I \\
\hline 12 Gyr (1\%) & 38.70 & 37.44 & 36.59 & 35.78 \\
12 Gyr (10\%) & 36.20 & 34.94 & 34.09 & 33.28 \\
12 Gyr (100\%) & 33.70 & 32.44 & 31.59 & 30.78 \\
14 Gyr (1\%) & 40.45 & 39.12 & 38.22 & 37.38 \\
14 Gyr (10\%) & 37.95 & 36.62 & 35.72 & 34.88 \\
14 Gyr (100\%) & 35.45 & 34.12 & 33.22 & 32.38 \\
\hline
\end{tabular}

undetectable. More metal-rich halos would be somewhat brighter because the main-sequence lifetime of stars increases with stellar metallicity, but still well below any realistically observable limit.

\section{Summary}

We have explored the photometric ramifications of ascribing a significant fraction of a galactic halo's dynamical mass to a population of old, faint, white dwarfs. Using our population synthesis package, we show that while such an hypothesis is essentially impossible to rule out using surface brightness arguments for nearby (old) halos, passive evolution of the progenitors of any large, putative, halo white dwarf population would make the presentday halos 100-500 times brighter at redshifts $1 \lesssim z \lesssim 3$ than halos evolving passively under standard stellar evolution arguments (i.e. a Salpeter-like IMF).

Making one-to-one comparisons between single objects such as the Milky Way and potentially quite different objects at high-redshift can be dangerous; what we wish to end with is simply a note drawing attention to, for example, the existence of passively-evolving $\mathrm{L}^{*}$ galaxies at high redshift which individually are consistent with passive evolution of normal stellar populations and normal IMFs (e.g. Waddington et al. 2002), i.e. the stellar populations therein are less than two magnitudes brighter than today (under reasonable assumptions of mass, etc.), opposed to being more than four to five magnitudes brighter than expected if these galaxies were dominated dynamically by white dwarf precursors. A safer comparison is to model the ensemble of the population, using parameters such as extragalactic background light and deep number counts, both of which are also inconsistent with the white dwarf scenario (Charlot \& Silk 1995; Madau \& Pozzetti 2000).

\section{Acknowledgements}

We wish to acknowledge the Australian Research Council, through its Discovery Project and Linkage International schemes, for ongoing financial support. We are grateful to the anonymous referees for their detailed reports that helped us improve this paper. We also thank Gilles Chabrier for stimulating our pursuit of this result.

\section{References}

Adams, F. C., \& Laughlin, G. 1996, ApJ, 468, 586

Afonso, C., et al. 2003, A\&A, 400, 951

Alcock, C., et al. 2000, ApJ, 542, 281

Bothun, G. D., Impey, C. D., Malin, D. F., \& Mould, J. R. 1987, AJ, 94, 23

Brook, C., Kawata, D., \& Gibson, B. K. 2003, MNRAS, 343, 913

Chabrier, G. 2001, in White Dwarfs as Dark Matter, eds.

H. B. Richer, \& B. K. Gibson, http://www.astro.ubc.ca/ WD_workshop/talks/

Chabrier, G., Segretain, L., \& Mera, D. 1996, ApJ, 468, L21

Charlot, S., \& Silk, J. 1995, ApJ, 445, 124

Di Stefano, R. 2000, ApJ, 541, 587

Durrell, P. R., Harris, W. E., \& Pritchet, C. J. 2001, AJ, 121, 2557

Fich, M., \& Tremaine, S. 1991, ARA\&A, 29, 409

Gibson, B. K., \& Mould, J. R. 1997, ApJ, 482, 98

Gould, A., Flynn, C., \& Bahcall, J. N. 1998, ApJ, 503, 798

Hansen, B. M. S. 1998, Nature, 394, 860

Kim, Y.-C., Demarque, P., Yi, S. K., \& Alexander, D. R. 2002, ApJS, 143, 499

Kuijken, K., \& Gilmore, G. 1991, ApJ, 367, L9

Larson, R. B. 1986, MNRAS, 218, 409

Lasserre, T., et al. 2000, A\&A, 355, L39

Lee, H.-c., Lee, Y.-W., \& Gibson, B. K. 2002, AJ, 124, 2664

Lee, H.-c., Yoon, S.-J., \& Lee, Y.-W. 2000, AJ, 120, 998

Lejeune, T., Cuisinier, F., \& Buser, R. 1998, A\&AS, 130, 65

Madau, P., \& Pozzetti, L. 2000, MNRAS, 312, L9

Preston, G. W., Shectman, S. A., \& Beers, T. C. 1991, ApJ, 375, 121

Ryan, S. G., \& Norris, J. E. 1991, AJ, 101, 1865

Richer, H. B., Hansen, B., Limongi, M., Chieffi, A., Straniero, O., \& Fahlman, G. G. 2000, ApJ, 529, 318

Rubin, V. C., Ford Jr, W. K., \& Thonnard, N. 1980, ApJ, 238, 471

Ryu, D., Olive, K. A., \& Silk, J. 1990, ApJ, 353, 81

Sahu, K. C., \& Sahu, M. S. 1998, ApJ, 508, L147

Salpeter, E. E. 1955, ApJ, 121, 161

Smecker, T. A., \& Wyse, R. 1991, ApJ, 372, 448

Waddington, I., et al. 2002, MNRAS, 336, 1342

Yi, S. 2003, ApJ, 582, 202

Yi, S., Demarque, P., \& Kim, Y.-C. 1997, ApJ, 482, 677 